\title{
Persistent Aura with Small Occipital Cortical Infarction: Implications for Migraine Pathophysiology
}

\author{
Sam Thissen Peter J. Koehler \\ Department of Neurology, Atrium Medical Centre, Heerlen, The Netherlands
}

\section{Key Words}

Migraine with aura - Persistent migraine aura - Inhibitory GABAergic network cells · Cortical hyperexcitability $\cdot$ Intracortical disinhibition hypothesis

\begin{abstract}
Objective: The pathophysiology of migraine with aura is thought to be related to cortical spreading depression and cortical hypersensitivity, in which inhibitory interneurons may play a role. Persistent migraine aura (PMA) without infarction is defined as auras that last longer than 1 week in the absence of infarction. We describe a case of persistent aura with a small occipital cortical infarction and discuss implications of this case and PMA for pathophysiological concepts of migrainous auras. Methods: We present a case and discuss the implications for pathophysiological concepts. Results: The case presented cannot be diagnosed as PMA as the patient was found to have an occipital cortical infarction with hypoactivity on fluorodeoxyglucose-positron emission tomography. Nevertheless, the patient suffered from persistent aura (with infarction). We argue that the infarction may have been responsible for an increased imbalance in one of the primary visual cortex networks that was already hyperexcitable due to the migraine aura condition. Conclusion: PMA with occipital infarction has not been reported previously. We believe the findings of the present case and PMA cases reported in the past may support the intracortical disinhibition hypothesis in migraine.
\end{abstract}

(C) 2014 S. Karger AG, Basel

\section{Introduction}

Migraine auras (MA) are recurrent attacks of reversible focal neurological symptoms that typically spread in 5-20 min and each symptom does not last more than $60 \mathrm{~min}$. If these symptoms last longer - between $60 \mathrm{~min}$ and 1 week - they are called prolonged aura. The

Sam Thissen, MD

Department of Neurology, Atrium Medical Centre

PO Box 4446

NL-6401 CX Heerlen (The Netherlands)

E-Mail s.thissen@atriummc.nl 
Thissen and Koehler: Persistent Aura with Small Occipital Cortical Infarction: Implications for Migraine Pathophysiology

aura is thought to be associated with Leao's cortical spreading depression (CSD), and cortical hyperexcitability is thought to be an important pathophysiological concept in MA [1]. Persistent migraine aura (PMA) without infarction is a condition in which auras last longer than 1 week in the absence of radiological evidence of infarction [2]. We present an observation of PMA with infarction, which was also briefly mentioned in our recent review on the PMA literature [3], and discuss the implications of this finding in the light of current pathophysiological ideas with respect to MA as well as PMA.

\section{Case Presentation}

A 74-year-old woman had suffered from migraine with aura for many years. The attacks usually started with left-sided lightning flashes and coloured dots followed by unilateral throbbing headache. Over the past years, her migraine attacks became rarer. In April 2011, she visited our outpatient clinic with complaints of continuous visual disturbances that had started a few weeks before. For 2 months she had been experiencing continuous zigzag lines and lightning flashes in her left visual field, which were sometimes accompanied by flickering lights. The phenomena were similar to her typical left-sided visual MA, and she did not complain of headache or nausea. The patient provided informed consent to participate in the investigation.

Neurological and ophthalmological examination was normal as were laboratory workup and electroencephalography. An MRI scan of the brain showed a small paramedian infarction in the right occipital lobe (fig. 1a). It was more pronounced on a second MRI scan in which a perfusion-weighted series was included showing a global perfusion defect in the same area (fig. 1b). Fluorodeoxyglucose-positron emission tomography showed hypoactivity in the right occipital lobe (fig. 2). The patient was diagnosed with migrainous infarction and received aspirin (100 mg daily). For the persisting aura we prescribed acetazolamide (250 $\mathrm{mg}$ daily), sodium valproate (1,000 $\mathrm{mg}$ daily) and nimodipine (240 mg daily) consecutively, but without any improvement of her complaints. She decided to stop taking any preventive drugs and at the latest follow-up 2 years after the start of the continuous aura, she stated that the aura had disappeared and that she only noticed some visual spots on the left side. Visual field examination, however, did not demonstrate any defect [3].

\section{Discussion}

\section{Cortical Hyperexcitability}

Our patient does not fulfill the criteria for PMA as she was found to have a small cortical ischaemic infarction in the right occipital lobe probably associated with it. The infarction was apparently too small to lead to homonymous hemianopia and instead produced positive symptoms for almost 2 years, finally leaving her with a non-detectable small negative spot in her left visual field. We believe the combination of persistent aura with a small infarction is of interest. Several studies have shown that the cerebral cortex in migraine patients displays an enhanced responsiveness to various external stimuli [4-8]. In migraine with aura, visual stimulus processing is believed to be disturbed to the extent that it is more difficult for these patients, as compared to matched controls, to suppress the perception of simple targets. The phenomenon fits into the concept of cortical neuronal hyperexcitability, which is thought to lead to CSD in certain conditions. It has been postulated as an important contributing factor 
Thissen and Koehler: Persistent Aura with Small Occipital Cortical Infarction: Implications for Migraine Pathophysiology

in the pathophysiology of migraine [9]. In PMA this cortical neuronal hyperexcitability is thought to be sustained, which could lead to reverberating CSD [3].

A possible mechanism for this presumed cortical hyperexcitability may be a lack of inhibitory control in the primary visual cortex (V1). In the human motor cortex, it has been shown that the cortical stimulation silent period - a measure of local cortical inhibition - is shortened in migraine, which is another possible indication pointing to deficient cortical inhibition [10]. Chronicle and Mulleners [11] argue that it is conceivable that repeated ischaemic or near-ischaemic reductions of regional cerebral blood flow in the V1 during the aura phase of migraine give rise to selective damage to the GABAergic inhibitory network of sparsely spinous stellate cells. These cells form a diffuse horizontal network in lamina IV of the V1. The possible ischaemic damage of these cells could be responsible for the cortical visual dysfunction shown to exist in MA patients by a number of investigators. Up to the present, this damage has not been visualised in studies using MRI because of its diffuse, ultrastructural and laminar nature [11].

Another theory for cortical hyperexcitability in migraine is that beside reduced inhibition, there is a low cortical preactivation. Coppola et al. [12] propose that reduced inhibition (possibly by diffuse damage to GABAergic inhibitory network cells as stated above) and low cortical preactivation due to thalamocortical hypoactivity may coexist. The latter can promote the former via reduction of lateral inhibition during the stimulus repetition. Eventually, both will lead to an increased cortical response to repeated stimuli, i.e. a lack of habituation. Further studies are needed to confirm these functional abnormalities.

\section{Cerebral Damage in Migraine with Aura}

It is well-known that MA, particularly in women, is associated with cerebral infarction and vascular complications in general. Cerebral damage in MA has been demonstrated in several studies; however, these concern white matter alterations in general [13]. Moreover, the majority of these alterations was found infratentorially. In MA, the presumed liability of CSD is already present. We argue that our patient's persisting positive symptoms following a migrainous infarction in the occipital lobe might be explained by cell dysfunction of a local inhibitory network in the right V1. The small infarction may have been responsible for an increased imbalance in one of the V1 networks that was already hyperexcitable due to the MA condition. It might be useful to do more imaging studies, not only longitudinally as proposed by Lipton and colleagues [13], but also with higher field powers as most studies were done with 1.5-tesla MRIs with a few exceptions (detecting grey matter alterations) [14]. After all, multiple sclerosis was considered a white matter disease until higher-fieldpower MRI studies revealed cortical alterations [15].

\section{Conclusion}

To our knowledge, this is the first observation of persistent aura (with positive phenomena) in migrainous infarction. We hypothesise that our patient's persisting positive symptoms following a migrainous infarction in the occipital lobe may be explained by cell dysfunction of an inhibitory network in the right V1. The small infarction may have been responsible for an increased imbalance in one of the V1 networks that was already hyperexcitable due to the MA condition. Damage to inhibitory GABAergic network cells in the occipital lobe in combination with low cortical preactivation through thalamocortical hypoactivity could give rise to important pathophysiological mechanisms, which lead to sustained cortical hyperexcitability in PMA, thereby resulting in reverberating CSD. More 
Thissen and Koehler: Persistent Aura with Small Occipital Cortical Infarction: Implications for Migraine Pathophysiology

imaging studies are needed, longitudinally and with higher field powers, to gather evidence for this theory and to detect damage in the V1 inhibitory networks.

\section{Disclosure Statement}

On behalf of all authors, the corresponding author states that there are no conflicts of interest.

\section{References}

1 Charles AC, Baca SM: Cortical spreading depression and migraine. Nat Rev Neurol 2013;9:637-644.

12 Headache Classification Committee of the International Headache Society: The International Classification of Headache Disorders, 3rd edition (beta version). Cephalalgia 2013;33:629-808.

-3 Thissen S, Vos IG, Schreuder AHCML, Schreurs WMJ, Postma AA, Koehler PJ: Persistent migraine aura; new cases, a literature review and ideas about pathophysiology. Headache 2014, Epub ahead of print.

-4 Wray SH, Mijovic-Prelec D, Kosslyn SM: Visual processing in migraineurs. Brain 1995;118:25-35.

-5 Chronicle EP, Wilkins AJ, Coleston DM: Thresholds for detection of a target against a background grating suggest visual dysfunction in migraine with aura but not migraine without aura. Cephalalgia 1995;15:117122.

-6 Aurora SK, Ahmad BK, Welch KMA, Bhardhawaj P, Ramadan NM: Transcranial magnetic stimulation confirms hyperexcitability of occipital cortex in migraine. Neurology 1998;50:1111-1114.

7 Aguggia M, Zibetti M, Febbraro A, Mutani R: Transcranial magnetic stimulation in migraine with aura: further evidence of occipital cortex hyperexcitability (abstract). Cephalalgia 1999;19:465.

$>8$ Mulleners WM, Chronicle EP, Palmer JE, Koehler PJ, Vredeveld JW: Suppression of perception in migraine: evidence for reduced inhibition in the visual cortex. Neurology 2001;56:178-183.

$>9$ Welch KMA, Barkley GL, Tepley N, Ramadan NM: Central neurogenic mechanisms of migraine. Neurology 1993;43(suppl 3):S21-S25.

10 Aurora SK, Ahmad BK, Al-Sayed F, Welch KMA: Cortical stimulation silent period is shortened in migraine with aura (abstract). Neurology 1998;50(suppl 4):A351-A352.

11 Chronicle E, Mulleners W: Might migraine damage the brain? Cephalalgia 1994;14:415-418.

12 Coppola G, Parisi V, Di Lorenzo C, Serrao M, Magis D, Schoenen J, Pierelli F: Lateral inhibition in visual cortex of migraine patients between attacks. J Headache Pain 2013;14:20.

13 Bashir A, Lipton RB, Ashina S, Ashina M: Migraine and structural changes in the brain: a systematic review and meta-analysis. Neurology 2013;8:1260-1268.

14 Rocca MA, Ceccarelli A, Falini A, et al: Brain grey matter changes in migraine patients with T2-visible lesions: a 3-T MRI study. Stroke 2006;37:1765-1770.

15 Ceccarelli A, Bakshi R, Neema M: MRI in multiple sclerosis: a review of the current literature. Curr Opin Neurol 2012;25:402-409. 


\section{Case Reports in Neurology}

\begin{tabular}{l|l}
\hline Case Rep Neurol 2014;6:217-221 \\
\hline DOI: $10.1159 / 000366409$ & $\begin{array}{l}\text { C 2014 S. Karger AG, Basel } \\
\text { www.karger.com/crn }\end{array}$ \\
\hline
\end{tabular}

Thissen and Koehler: Persistent Aura with Small Occipital Cortical Infarction: Implications for Migraine Pathophysiology

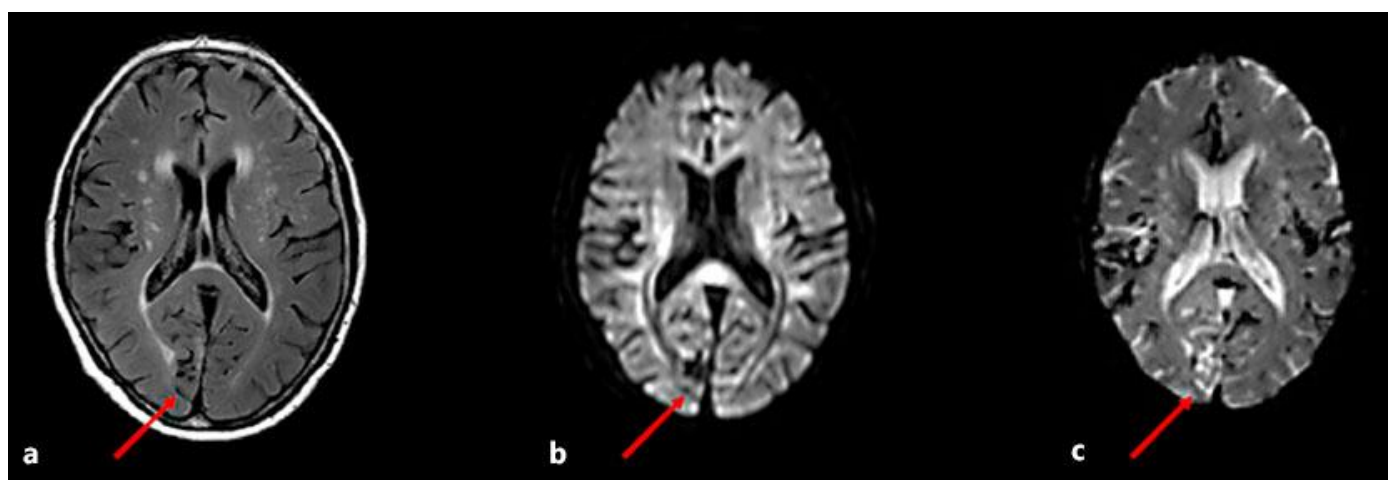

Fig. 1. a MR FLAIR sequence showing a small area of cortical damage in the paramedian right occipital lobe. b MR diffusion-weighted imaging sequence showing the same cortical damage in the right occipital lobe without diffusion abnormalities. c Perfusion MRI showing a global perfusion defect in the same area in the right occipital lobe.

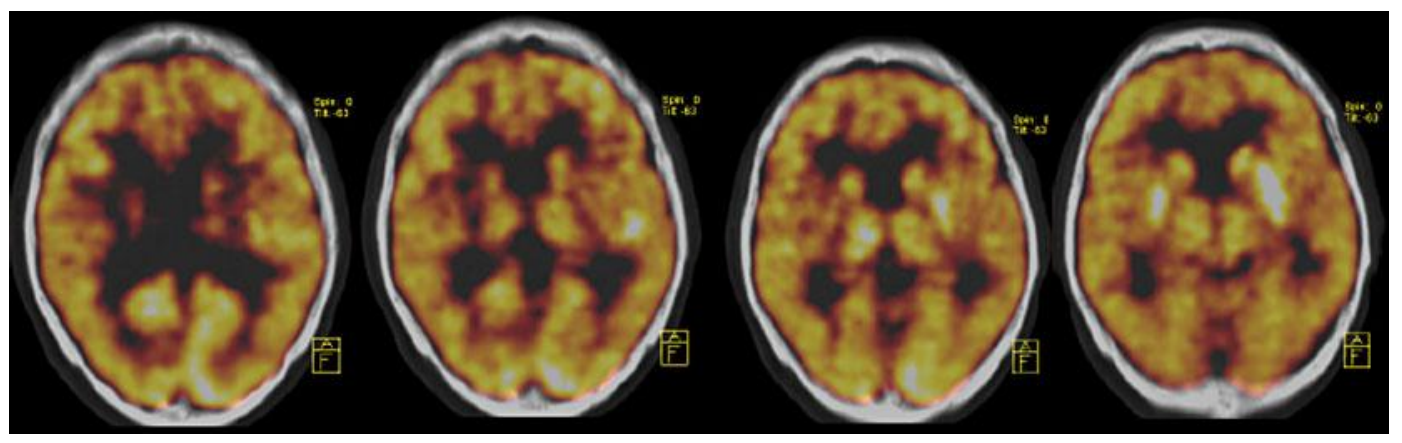

Fig. 2. Fluorodeoxyglucose-positron emission tomography showing hypoactivity in the right occipital lobe. 